\title{
QUALIDADE DA ÁGUA do RIO DAS PEDRAS, OESTE DO PARANÁ, UTILIZANDO MACROINVERTEBRADOS BENTÔNICOS COMO BIOINDICADORES
}

\author{
Marcelo Bevilacqua Remor ${ }^{1}$ \\ Mário Hermoso ${ }^{2}$ \\ Luciano Fabris Sgarbi ${ }^{3}$ \\ Tânia Mari Vicentini Prestes ${ }^{4}$ \\ Carla Daniela Câmara ${ }^{4}$ \\ Kathleen Jeniffer Model ${ }^{1}$
}

REMOR, M. B.; HERMOSO, M.; SGARBI, L. F.; PRESTES, T. M. V.; CÂMARA, C. D.; MODEL, K. J. Qualidade da água do rio das Pedras, oeste do Paraná, utilizando macroinvertebrados bentônicos como bioindicadores. Arq. Ciênc. Vet. Zool. UNIPAR, Umuarama, v. 17, n. 2, p. 121-129, abr./jun. 2014.

RESUMO: Os macroinvertebrados bentônicos são amplamente utilizados como bioindicadores de qualidade da água em todo o mundo, devido, as suas características fisiológicas e morfológicas. O objetivo do estudo foi avaliar a qualidade da água do rio das Pedras,no município de Matelândia, Oeste do Estado do Paraná, na estação seca, utilizando-se de macroinvertebrados bentônicos como bioindicador, e identificar o tempo ideal de incubação dos litter bags. Sessenta litter bags foram confeccionados com folhas de árvores nativas provenientes da floresta ripária e incubadosno rio das Pedras. Oito litter bags foram coletados nos intervalos de três, sete,14, 28, 35 e 42 dias. Os macroinvertebrados bentônicos foram identificados em nível de família e foi aplicado o índice Biological Monitoring Working Party Score System. Com este estudo concluiu-se que o rio das Pedras possui águas não poluídas, demonstrando que o sistema é perceptivelmente não alterado. O tempo de incubação dos litter bags para obtenção do resultado quanto à avaliação da qualidade de água pelo índice Biological Monitoring Working Party Score System foi de sete dias e para analisar a estrutura da comunidade de macroinvertebrados bentônicos foi de 28 dias.

PALAVRAS-CHAVE: Colonização. Litter bags. Tempo de incubação.

\section{WATER QUALITY AT RIO DAS PEDRAS, WESTERN PARANÁ, USING BENTHIC MACROINVERTEBRATES AS INDICATORS}

\begin{abstract}
Benthic macroinvertebrates are widely used as bioindicators of water quality due to their physiological and morphological characteristics. The aim of this study was to evaluate the water quality at Rio das Pedras, in the city of Matelândia, Western Paraná, in the dry season, using benthic macroinvertebrates as bioindicators, and to identify the optimal time for the incubation of litter bags. Sixty litter bags were prepared with leaves from native trees from the riparian forest and incubated in Rio das Pedras river. Eight litter bags were collected at intervals of 3, 7, 14, 28, 35 and 42 days. The benthic macroinvertebrates were identified at the family level and the Biological Monitoring Working Party Score System index was applied. With this study, it can be concluded that the Rio das Pedras river has unpolluted waters, demonstrating that the system has hardly been changed. The litter bag incubation period in order to obtain the results for evaluating the water quality index for the Biological Monitoring Working Party Score System was of seven days, and the period to analyze the structure of the benthic macroinvertebrate community was of 28 days.
\end{abstract}

KEY WORDS: Colonization. Litter bags. Incubation time.

\section{CALIDAD DE LAS AGUAS DEL RIO DAS PEDRAS, OESTE DEL PARANÁ, UTILIZANDO MACROINVERTEBRADOS BENTÓNICOS COMO BIOINDICADORES}

RESUMEN: Los macroinvertebrados bentónicos son ampliamente utilizados como bioindicadores de calidad del agua en todo el mundo, debido sus características fisiológicas y morfológicas. El objetivo de este estudio ha sido evaluar la calidad de las aguas del Rio das Pedras, en el municipio de Matelândia, oeste del Estado de Paraná, en la estación seca, utilizándose de macroinvertebrados bentónicos como bioindicador, e identificar el tiempo ideal de incubación de los litter bags. Sesenta litter bags han sido confeccionados con hojas de árboles nativas procedentes de la floresta ribera e incubados en el Rio das Pedras. Ocho litter bags fueron recogidos en intervalos de tres, siete, catorce, veintiocho, treinta y cinco y cuarenta y dos días. Los

\footnotetext{
OI: https://doi.org/10.25110/arqvet.v17i2.2014.4930

${ }^{1}$ Universidade Estadual do Oeste do Paraná - UNIOESTE, Pós-graduação em Engenharia Agricola. Rua Universitária n. 2069, Jardim Universitário, Cascavel, PR.CEP: 85.819-110.remor_@hotmail.com,kathleen05jmodel@gmail.com;

${ }^{2}$ Faculdade Assis Gurgacz - FAG, Pós-graduação em Auditoria, Perícia e Gestão Ambiental. Avenida das Torres n. 500, Santa Cruz, Cascavel, PR. CEP: 85.806-095. hermoso_mario@hotmail.com;

${ }^{3}$ Universidade Federal de Goiás - UFG. Pós-graduação em Ecologia e Evolução. ICB I, Sala 222, Goiânia, GO. CEP: 74001-970. luciano.f.sgarbi@gmail. com;

${ }^{4}$ Universidade Tecnológica Federal do Paraná - UTFPR. Curso de Tecnologia em Gestão Ambiental. Avenida Brasil, 4232, Parque Independência, Medianeira, PR. CEP: 85884-000. taniaprestes@hotmail.com, camara@utfpr.edu.br.
} 
macroinvertebrados bentónicos fueron identificados en nivel de familia y aplicado el índice Biological Monitoring Working Party Score System. Con esta investigación se ha concluido que el Rio das Pedras no tiene aguas contaminadas, demostrando que el sistema no es alterado. El tiempo de incubación de los litter bags para obtención del resultado cuanto a la evaluación de la calidad del agua por el índice Biological Monitoring Working Party Score System fue de siete días, y para analizar la estructura de la comunidad de macroinvertebrados bentónicos fue de 28 días.

PALABRAS CLAVE: Colonización. Litter bags. Tiempo de incubación.

\section{Introdução}

Os ecossistemas aquáticos têm sido alterados de maneira significativa, devido a múltiplos impactos ambientais indesejáveis, resultantes da influência direta ou indireta das atividades antrópicas (CALLISTO; MORETTI; GOULART, 2001; BORTOLUZZI et al., 2007). Entre esses pode-se citar a retirada da floresta ripária, construção de barragens e represas, uso inadequado do solo próximo a mananciais, atividades agropecuárias, lançamento de efluentes domésticos e industriais (CALLISTO; GONÇALVES; MORENO, 2005). Como consequência, vêm aumentando os casos de enfermidades por doenças de veiculação hídrica, além da perda do valor estético e recreativo desses ambientes (CORGOSINHO et al., 2004).

A identificação e quantificação desses impactos pode ser realizada por meio de bioindicadores. As comunidades biológicas refletem a integridade ecológica dos ecossistemas, integrando os efeitos dos diferentes agentes impactantes e fornecendo uma medida agregada dos impactos (BARBOUR et al., 1999). Os macroinvertebrados bentônicos vivem no fundo de corpos d'água continentais, dentre eles predominam as larvas de insetos aquáticos, oligoquetas, moluscos, crustáceos, entre outros com tamanhos de corpo maiores que 0,5 mm (ESTEVES, 1998; CALLISTO; GONÇALVES; MORENO, 2005). Estes organismos são eficientes para a avaliação e monitoramento de impactos de atividades antrópicas em ecossistemas aquáticos continentais (GOULART; CALLISTO, 2003), principalmente porque em condições ambientais específicas, alguns grupos mais resistentes podem se tornar dominantes enquanto os mais sensíveis, raros ou ausentes (ABÍLIO et al., 2007). Além disso, apresentam características específicas que favorecem o seu uso como bioindicadores tais como: são encontrados em todos os ambientes aquáticos, possuem mobilidade limitada, ampla variedade de respostas aos estresses ambientais, existem espécies com ciclo longo que possibilitam a análise temporal das perturbações sobre a comunidade e a amostragem dos mesmos é simples obtendo-se com materiais de baixo custo (MORENO; CALLISTO, 2005; MONTEIRO et al., 2007).

Segundo Metcalfe (1989), o uso das respostas biológicas é vantajoso em comparação com medidas físicas e químicas para demonstrar a degradação ambiental. Análises físicas e químicas registram apenas o momento da coleta, necessitando assim de mais repetições de análises para a realização de um monitoramento temporal eficiente.

A amostragem de macroinvertebrados pode ser realizada com auxílio de litter bags, pois este é uma ferramenta simples para avaliar a qualidade biológica de ecossistemas lóticos e lênticos baseada na colonização natural dos organismos. São construídos confeccionando-se uma "bolsa" (com tela de material plástico ou metálico, a malha pode variar de 0,15 a $30 \mathrm{~mm}$ ) esta pode ser recheada com folhas de árvores, pedras, plástico ou cerâmica. Posteriormente são dispostos no ambiente, para serem colonizadas pelos organismos e retirados em datas predeterminadas (ROSENBERG; RESH, 1982). O objetivo do estudo foi avaliar a qualidade da água do rio das Pedras na estação seca, utilizando-se de macroinvertebrados bentônicos como bioindicador, e identificar o tempo ideal de incubação dos litter bags.

\section{Material e Métodos}

Área de estudo: O estudo foi conduzido nos meses de maio e junho de 2009, no município de Matelândia, região oeste do Paraná, coordenadas 259'10" S; 5356'50" W, altitude de $590 \mathrm{~m}$. O clima da região segundo a classificação de Köeppen é do tipo subtropical mesotérmico superúmido, com precipitação média anual de $1800 \mathrm{~mm}$, verões quentes e tendência de concentração das chuvas nos meses de verão. A temperatura média é de $20^{\circ} \mathrm{C}$ e umidade relativa do ar em média de 75\% (CAVIGLIONE et al., 2000). A vegetação nativa é classificada como Floresta Estacional Semidecidual.

O Rio das Pedras é um rio de segunda ordem e possui as seguintes características: aproximadamente cinco quilômetros de extensão da nascente até a foz no rio Xaxim. A área onde foi conduzido o estudo fica a aproximadamente um quilômetro da foz, possui largura de quatro metros e profundidade média de 0,4 metros. A floresta ripária é conservada e composta basicamente por espécies nativas e com largura média de 25 metros em cada margem.

Condução do estudo: As folhas das plantas nativas foram coletadas na vegetação ripária do riacho, foram identificadas e secas em estufas de ventilação forçada, a temperatura de $60^{\circ} \mathrm{C}$ por 24 horas.

Sessenta litter bags foram confeccionados em tela de polietileno de alta densidade com malha de $20 \mathrm{~mm}$, costurados com linha de nylon, formando embalagem de 250x200 $\mathrm{mm}$. Posteriormente foram introduzidos cinco gramas das folhas secas em cada litter bag. As plantas nativas utilizadas na confecção dos litter bags estão indicadas na Tabela 1.

Tabela 1: Espécies de plantas utilizadas na confecção do litter bag

\begin{tabular}{|c|c|c|}
\hline $\begin{array}{c}\text { Nome } \\
\text { Popular }\end{array}$ & Família & Nome Científico \\
\hline Cedro & Meliaceae & $\begin{array}{l}\text { Cedrella fissilis } \\
\text { VELLOZO }\end{array}$ \\
\hline Guabiroba & Myrtaceae & $\begin{array}{l}\text { Campomanesia xantho- } \\
\text { carpa } \mathrm{O} . \mathrm{BERG}\end{array}$ \\
\hline Açoita Cavalo & Tiliaceae & $\begin{array}{l}\text { Luehea divaricata } \\
\text { MARTIUS \& ZUCCA- } \\
\text { RINI }\end{array}$ \\
\hline Pata de vaca & Caesalpinaceae & $\begin{array}{l}\text { Bauhinia forficata } \\
\text { BENTH }\end{array}$ \\
\hline
\end{tabular}




\begin{tabular}{|c|c|c|}
\hline Ipê-amarelo & Bignoniaceae & $\begin{array}{l}\text { Tabebuia Alba } \\
\text { (CHAM.) SANDWITH }\end{array}$ \\
\hline gá-do-brejo & Mimosoideae & $\begin{array}{l}\text { Inga uruguensis } \\
\text { HOOKER \& ARNOTT }\end{array}$ \\
\hline rva-mate & Aquifoliaceae & $\begin{array}{l}\text { Ilex paraguariensis } \\
\text { SAINT HILAIRE }\end{array}$ \\
\hline anela guaicá & Lauraceae & $\begin{array}{l}\text { Ocotea puberula } \\
\text { (RICH.) NEES }\end{array}$ \\
\hline eroba rosa & Apocynace & $\begin{array}{l}\text { Aspidosperma polyneu- } \\
\text { ron MUELL. ARG. }\end{array}$ \\
\hline
\end{tabular}

Os litter bags, lacrados e numerados foram transportados em sacos plásticos, incubados no leito do riacho utilizando-se pedras e cordas de nylon para sua fixação. Os litter bags foram dispostos ao longo do eixo longitudinal e transversal do riacho buscando a heterogeneidade do ambiente (Figura 1).

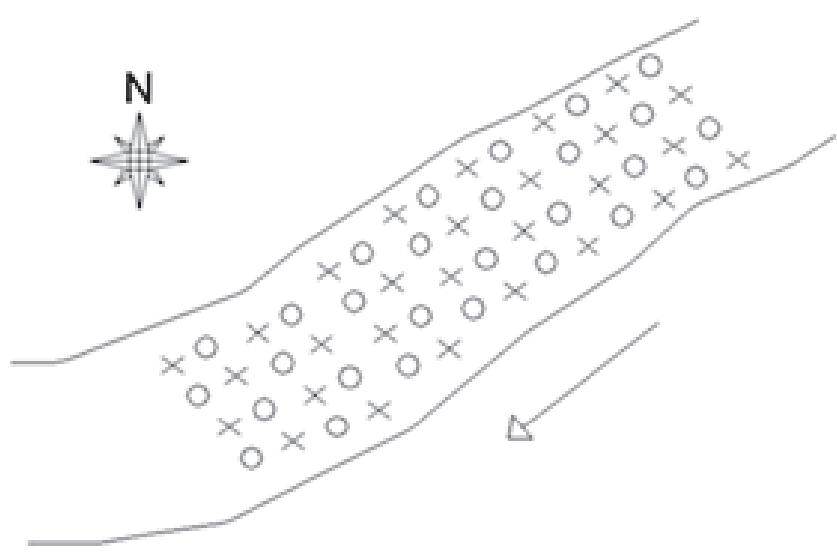

Figura 1: Disposição dos litter bags no leito do riacho. A seta indica o fluxo da correnteza.

Oito litter bags foram coletados nos intervalos de três, sete, 14, 28, 35, e 42 dias de incubação. Os litter bags foram retirados do riacho usando coletor de macroinvertebrados (rede D) de malha $0,25 \mathrm{~mm}$, acondicionados em sacos plásticos e transportados até o laboratório.

No laboratório foi realizada a triagem do substrato em conjunto de peneiras de $1 \mathrm{~mm}, 0,50 \mathrm{~mm}$ e $0,25 \mathrm{~mm}$ para separar as folhas dos macroinvertebrados. Os indivíduos encontrados foram conservados em álcool $70 \%$ e posteriormente identificados até nível de família, quando possível, com auxílio de microscópio estereoscópico e as chaves de identificação de McCafferty e Provonsha (1981) e Fernádez e Domínguez (2001).

A classificação dos organismos quanto à tolerância à poluição foi realizada com base no índice Biological Monitoring Working Party Score System (BMWP) adaptado por Loyola, (2000) (Anexo 1). Este método utiliza identificação dos organismos bentônicos ao nível taxonômico de família e estabelece uma pontuação para cada grupo com base na sua tolerância ao impacto, os valores variam entre um e 10 e são atribuídos de acordo com a sensibilidade das espécies a poluentes orgânicos. Famílias sensíveis a baixos níveis de poluentes recebem valores mais altos, enquanto famílias tolerantes recebem valores mais baixos (JUNQUEIRA et al., 2000). Os escores são somados e comparados com o tabela do Anexo 2 para obtenção da qualidade da água do ambiente.

Análise dos dados: Os índices descritivos da comunidade como número de táxons, número de indivíduos, dominância, diversidade e dominância de Simpson, diversidade de Shanon-Wiener, diversidade de Margalef e Equitabilidade foram calculados por meio de rotinas implementadas no programa PAST 2.15 (HAMMER et al., 2001). As demais análises estatísticas foram realizadas por meio de rotinas implementadas no programa R (R CORE TEAM, 2013).

Os dados obtidos foram transformados sob raiz quadrada obtendo-se assim simetria e curtose para realização das análises, sendo que os dados apresentados nas tabelas e figuras são os originais.

A análise Non-Metric Multidimencional Scaling (NMDS) entre os tempos de incubação foi realizada seguindo o protocolo de McCune e Grace (2002). A distância de Sorensen foi utilizada para mensuração da distância da matriz. A análise foi rodada no piloto automático com a semente 1840 , a velocidade lenta e o critério de estabilização do stress $=0,0000009$.

A análise de variância múltipla (MANOVA), análise de variância (ANOVA) e o teste de comparação de médias de Tukey entre os táxons encontrados e os tempos de incubação foram consideradas significativas quando o p-valor encontrado foi menor que 0,05 .

\section{Resultados e Discussão}

No estudo foram encontrados 4083 espécimes de macroinvertebrados bentônicos, representantes de 30 táxons (Tabela 2). Deste montante $2731(66,7 \%)$ são representantes da família Chironomidae, $248(6,1 \%)$ representantes da família Elmidae e 191 (4,7\%) representantes da família Simullidae. O número de táxons encontrados é similar ao estudo de Moretti et al. (2007) porém o número de indivíduos foi duas vezes maior. Gonçalves Jr. et al. (2012) constatou que as famílias Chironomidae e Elmidae também apresentaram densidade elevadas. Ressalta-se que a família Chironomidae normalmente é numericamente dominante em ambientes dulciaquícolas nas diferentes regiões climáticas (ESTEVES, 1998). 
Tabela 2: Táxons e números de indivíduos encontrados nos litter bags e valor do índice BMWP respectivos.

\begin{tabular}{|c|c|c|c|c|}
\hline Classe & Ordem & Família & $\mathrm{N}$ & BMWP \\
\hline \multirow{25}{*}{ Insecta } & \multirow{5}{*}{ Ephemeroptera } & Baetidae & 108 & 4 \\
\hline & & Caenidae & 156 & 4 \\
\hline & & Euthyplociidae & 9 & 8 \\
\hline & & Leptophlebiidae & 155 & 10 \\
\hline & & Leptohyphidae & 20 & 6 \\
\hline & \multirow{5}{*}{ Trichoptera } & Calamoceratidae & 60 & 10 \\
\hline & & Hydropsychidae & 62 & 5 \\
\hline & & Leptoceridae & 18 & 10 \\
\hline & & Odontoceridae & 1 & 10 \\
\hline & & Philopotamidae & 19 & 8 \\
\hline & \multirow[t]{4}{*}{ Odonata } & Calopterygidae & 47 & 8 \\
\hline & & Coenagrionidae & 10 & 6 \\
\hline & & Gomphidae & 1 & 8 \\
\hline & & Megapodagrionidae & 18 & 10 \\
\hline & \multirow{4}{*}{ Coleoptara } & Dytiscidae & 1 & 3 \\
\hline & & Elmidae & 248 & 5 \\
\hline & & Gyrinidae & 14 & 3 \\
\hline & & Psephenidae & 1 & 7 \\
\hline & \multirow{2}{*}{ Plecoptera } & Gripopterygidae & 1 & 7 \\
\hline & & Perlidae & 44 & 10 \\
\hline & \multirow{5}{*}{ Diptera } & Chironomidae & 2731 & 2 \\
\hline & & Empididae & 5 & 4 \\
\hline & & Psychodidae & 1 & 4 \\
\hline & & Simuliidae & 191 & 5 \\
\hline & & Tabanidae & 1 & 4 \\
\hline Crustacea & Decapoda & - & 47 & 6 \\
\hline Bivalvia & - & - & 95 & 4 \\
\hline Gastropoda & - & - & 3 & 3 \\
\hline Oligochaeta & - & - & 15 & 1 \\
\hline Hirudinea & - & - & 1 & 3 \\
\hline
\end{tabular}

N: Número de indivíduos encontrado; BMWP: escore do índice BMWP respectivo ao táxon.

Segundo o índice BMWP (Tabela 3) demonstra que a qualidade da água do rio das Pedras é boa, com águas não poluídas e um sistema perceptivelmente não alterado. Isso é condizente com as condições ambientais de sua floresta ri- pária, que é bem preservada, fazendo com que as atividades antrópicas realizadas em sua microbacia tenham efeito praticamente nulo sobre a comunidade de macroinvertebrados bentônicos (ROMANUK; LEVINGS, 2003).

Tabela 3: Índices descritivos da comunidade e índice Biological Monitoring Working Party Score System (BMWP) ao longo do tempo de incubação.

\begin{tabular}{lllllllll}
\hline $\mathbf{T}$ & $\mathbf{S}$ & $\mathbf{N}$ & $\mathbf{D}$ & $\mathbf{D s}$ & $\mathbf{H}^{\prime}$ & $\boldsymbol{\alpha}$ & $\mathbf{J}$ & $\mathbf{B}$ \\
\hline $\mathbf{3}$ dias & 19 & 735 & 0,633 & 0,367 & 0,99 & 2,73 & 0,336 & 109 Aceitável \\
7 dias & 24 & 913 & 0,507 & 0,493 & 1,33 & 3,37 & 0,418 & 147 Boa \\
14 dias & 20 & 1182 & 0,612 & 0,388 & 1,05 & 2,69 & 0,351 & 134 Boa \\
28 dias & 21 & 628 & 0,245 & 0,755 & 1,98 & 3,10 & 0,649 & 125 Boa \\
35 dias & 23 & 344 & 0,348 & 0,652 & 1,69 & 3,77 & 0,541 & 128 Boa \\
42 dias & 14 & 217 & 0,313 & 0,687 & 1,74 & 2,42 & 0,659 & 82 Duvidosa \\
\hline Total & 30 & 4085 & 0,458 & 0,542 & 1,49 & 3,49 & 0,439 & 178 Ótima
\end{tabular}

T: Tempo de Incubação; S: Número de Táxons; N: Número de Indivíduos; D: Dominância; D: Diversidade e Dominância de Simpson; H': Diversidade de Shanon-Wiener; $\alpha$ : Diversidade de Margalef; J: Equitabilidade; B: Escore e qualidade da água segundo o índice BMWP. 
$\mathrm{Na}$ segunda e terceira coleta com sete e quatorze dias de incubação respectivamente, verificou-se os maiores escores do índice BMWP, embora os índices descritivos da comunidade demonstrem maior estabilidade da comunidade na colonização dos litter bags na quarta e a quinta coleta, com 28 e 35 dias de incubação respectivamente (Tabela 3). Esses resultados podem ser explicados segundo a teoria da sucessão ecológica degradativa descrita por Begon, Harper e Townsend (1996). Essa teoria sustenta que a colonização de substratos começa com algumas espécies generalistas, em seguida, espécies mais especializadas começam a colonizar até atingir o clímax. Após o clímax a diversidade da comunidade começa a decair, porque o substrato atinge avançado estado de degradação e não consegue sustentar a comunidade com nutrientes e energia.

Os manuais práticos de coleta de invertebrados bentônicos para biomonitoramento aconselham que os litter bags permaneçam incubados por um período de 42 a 56 dias (GIBBONS et al., 1993; APHA, 1998), porém, tem sido considerado inadequado por alguns autores (MEIER et al., 1979). Segundo Rosenberg e Resh (1982) e Mackay (1992), o tempo ideal de incubação depende do desenho amostral, da estação do ano, da localização geográfica e a medida de equilíbrio considerada. É preciso levar em conta, entretanto, que períodos muito longos de exposição aumentam a probabilidade de perda por vandalismo e/ou inundações (CLEMENTS et al., 1989).

O melhor tempo de incubação dos litter bags para aplicação do índice BMWP foi de sete dias visto que apresentou o maior escore e possui o maior número de táxons encontrados. Na literatura, quando apenas o número de táxons foi utilizado como critério para medir equilíbrio e definir o tempo ideal de colonização, esse período pode ser rápido (quatro a seis dias) (TOWNSEND; HILDREW, 1976; LAKE; DOEG, 1985), porém, frequentemente, varia entre quatro a 28 dias (WISE; MOLES, 1979; ROSENBERG; RESH, 1982; MACKAY, 1992). Entretanto o melhor tempo de incubação para avaliar a estrutura da comunidade de macroinvertebrados bentônicos foi de 28 dias, pois esse apresenta os maiores valores de diversidade e equitablididade, consequentemente menor valor de dominância, demonstrando que a comunidade atingiu o seu equilíbrio. Este resultado é semelhante ao obtido por Boothroyd e Dickie (1989) e Kuhlmann et al. (2003).

Os táxons Dytiscidae, Gomphidae, Gripopterigidae, Hirudínea, Odontoceridae, Psephenidae, Psychodidae e Tabanidae foram excluídos das análises de NMDS, MANOVA e ANOVA, pois apresentavam comportamento de outliers (amostrado somente um indivíduo), prejudicando a confiabilidade e as inferências das análises.

O teste de Monte Carlo da análise NMDS recomendou analisar dois eixos, demonstrando que os resultados observados não podem ser explicados apenas pelo acaso $(\mathrm{p}=0,0040)$. O stress final foi de 4,87 mostrando que a qualidade do ajuste é excelente, com 64 interações e final estável, permitindo a realização de inferências confiáveis sobre a análise.

$\mathrm{Na}$ análise NMDS (Figura 2) foram interpretados dois eixos significativos, que demonstram a composição da comunidade de macroinvertebrados bentônicos em função dos tempos de incubação distintos.
A MANOVA entre os táxons e os tempos de incubação demonstra que existe variabilidade na abundância de alguns táxons em relação ao tempo de incubação (teste de Wilks $=0,000009$ e $\mathrm{p}=0,000009)$.

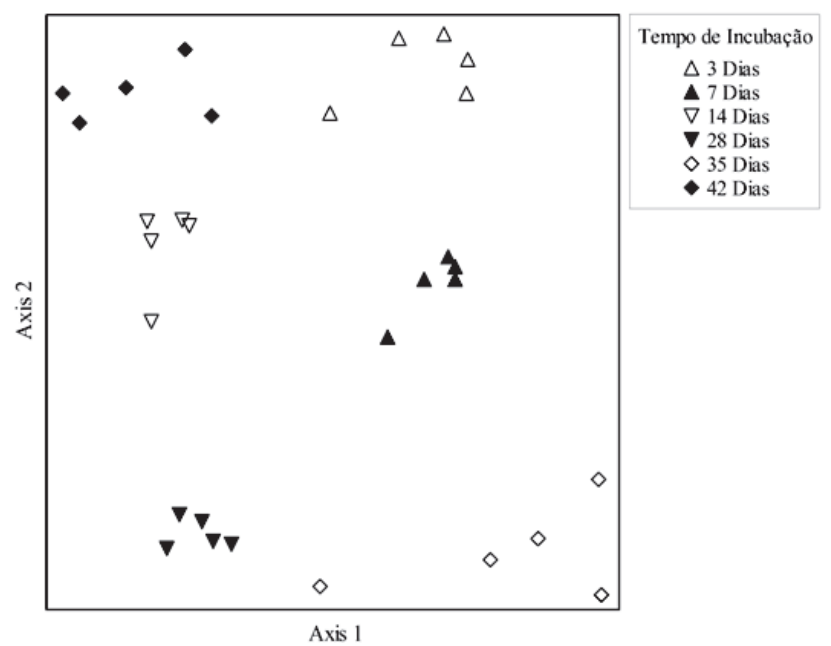

Figura 2: Análise Non-Metric Multidimensional Scaling entre os tempos de incubação.

AANOVA mostra que a abundância dos táxons Baetidae, Bivalvia, Caenidae, Calamoceratidae, Calopterygidae, Chironomidae, Decapoda, Elmidae, Empididae, Euthyplociidae, Gastropoda, Gyrinidae, Hydropsychidae, Leptoceridae, Leptohyphidae Leptophlebiidae, Megapodagrionidae, Oligochaeta, Perlidae, Philopotamidae, Simuliidae diferiu significativamente entre os tempos de incubação. Entretanto a abundância dos táxons coenagrionidae $(\mathrm{p}=0,26)$ e empididea $(p=0,59)$ não variou em função do tempo de incubação, pois estes estão distribuídos de modo uniforme entre os tempos.

Os táxons Gyrinidae, Perlidae, Philopotamidae e Simuliidade foram mais abundantes no período de sete dias de incubação (Figura 3). Os táxons Baetidae, Bivalvia, Caenidae, Calamoceratidae, Calopterygidae, Chironomidae, Decapoda, Leptoceridae e Megapodagrionidae, foram encontrados em maior número com 14 dias de incubação e os táxons Elmidae, Empididae, Euthyplociidae, Leptohyphidae, Leptophlebiidae, Hydropsychidae, foram mais abundantes no período de 28 dias de incubação. O táxon Oligochaeta, atingiu maior abundância com 42 dias de incubação. 

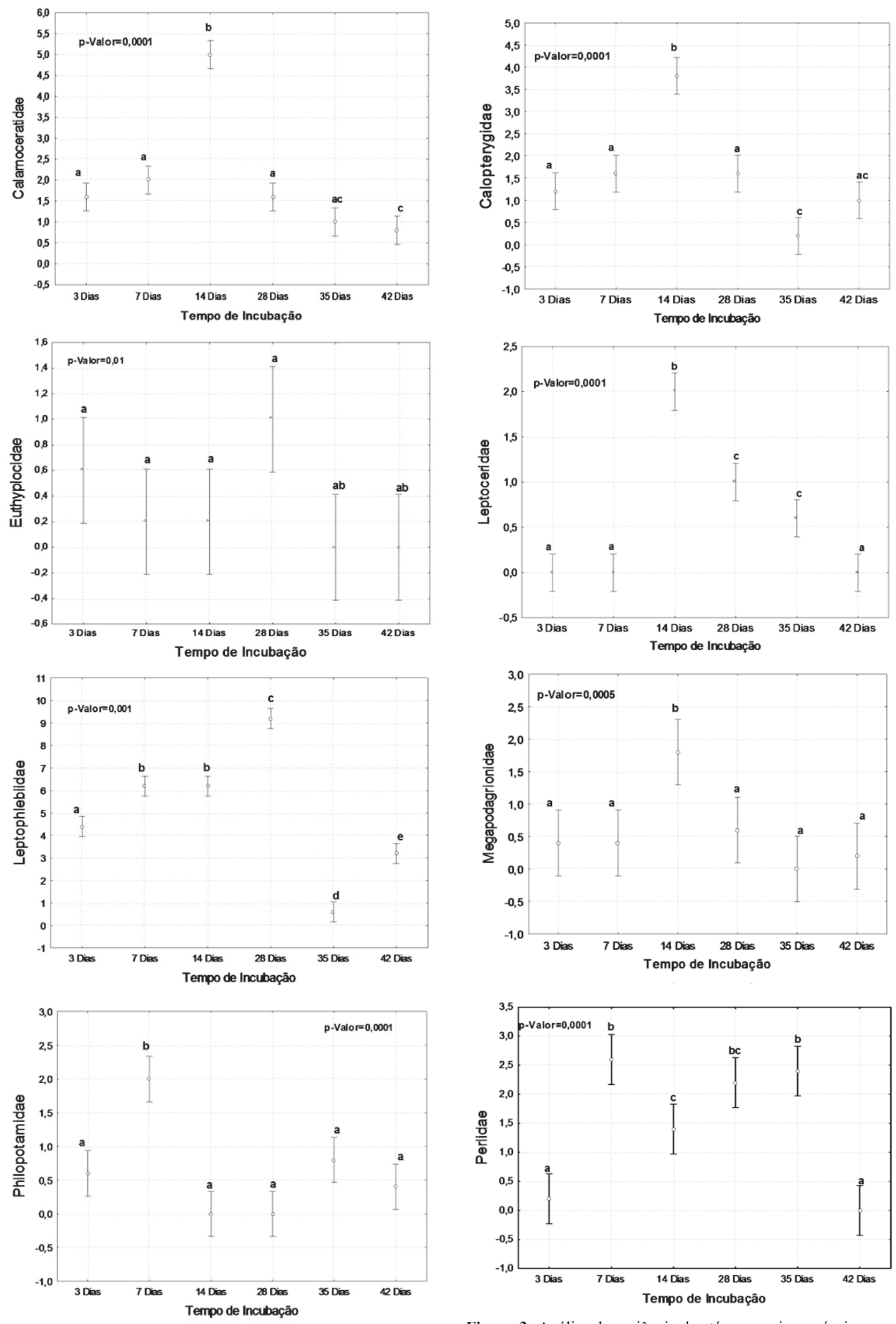

Figura 3: Análise de variância dos táxons mais sensíveis em relação ao tempo de incubação. 
Conclusão

O rio das Pedras localizado no município de Matelândia, região oeste do Paraná, possui águas não poluídas demonstrando que o sistema é perceptivelmente não alterado.

O litter bag se mostrou eficiente como amostrador de macroinvertebrados bentônico para estudos de avaliação da qualidade da água de rios. O tempo de incubação dos litter bags para obtenção do resultado quanto à avaliação da qualidade de água pelo índice Biological Monitoring Working Party Score System é de sete dias e para analisar a estrutura da comunidade de macroinvertebrados bentônicos é de 28 dias.

\section{Referências}

ABÍLIO, F. J. P. et al. Macroinvertebrados bentônicos como bioindicadores de qualidade ambiental de corpos aquáticos da caatinga. Oecologia Brasiliensis, v. 11, n. 3, p. 397-409, 2007.

APHA. Standard methods for the examination of water and wastewater. 20 ed. New York: APHA/AWWA/WEF, 1998. $1067 \mathrm{p}$.

BARBOUR, M. T. et. al. Rapid bioassessment protocols for use in streams and wadeable rivers. Periphyton, benthic macroinvertebrates and fish. 2 ed. New York: USEPA, 1999. 178 p.

BEGON, M.; HARPER, J. L.; TOWNSEND, C. R. Ecology: individuals, populations and communities. 3. ed. Oxford: Blackwell Science, 1996. 1068 p.

BOOTHROYD, I. K. G.; DICKIE, B. N. Macroinvertebrate colonization of Perspex artificial substrates for use in biomonitoring studies. New Zealand Journal of Marine and Freshwater Research, v. 23, p. 467-478, 1989.

BORTOLUZZI, L. R. et. al. Anomuros do gênero Aegla no arroio Imbaá. Biodiversidade Pampeana, v. 5, n. 2, p. 9-13, 2007.

CALlisto, M., MORETTI, M., GOULART, M. D. C. Macroinvertebrados bentônicos como ferramenta para avaliar a saúde de riachos. Revista Brasileira de Recursos Hídricos, v. 6, p. 71-82, 2001.

CALLISTO, M.; GONÇALVES, J. F.; MORENO, P. Invertebrados aquáticos como bioindicadores. In: GOULART, E. M. A. (Ed). Navegando o Rio das Velhas das Minas aos Gerais, 2005.

CAVIGLIONE, J. H. et. al. Cartas climáticas do Paraná Londrina, IAPAR. 2000.

CLEMENTS, W. H. et. al. Colonization, variability, and use of substratum filled trays for biomonitoring benthic communities. Hydrobiologia, v. 173, p. 45-53, 1989.

CORGOSINHO, P. H. C. et. al. Diversidade de habitats e padrões de diversidade e abundância do bentos ao longo de um afluente do reservatório de Três Marias, MG. Arquivos do Instituto Biológico, v. 71, p. 227-232, 2004.

ESTEVES, F. A. Fundamentos de Limnologia. 2. ed. Rio de Janeiro: Interciência, 1998. 602 p.

FERNÁDEZ, H. R.; DOMÍNGUEZ, E. Guia para la detreminación de los artropodos bentónicos sudamericanos. San Miguel de Tucumán: Universidad Nacional de Tucumán, 2001. 282 p.

GIBBONS, W. N.; MUNN, M. D.; PAINE, M. D. Guidelines for monitoring benthos in freshwater environments. Report prepared for Environment Canada. North Vancouver: B.C. by EVS Consultants, 1993. $81 \mathrm{p}$.

GONÇALVES, JUNIOR. et. al. Invertebrate colonisation during leaf processing of native, exotic and artificial detritus in a tropical stream. Marine and Freshwater Research, v. 63, n. 5, p. 428-439, 2012.

GOULART, M. D.; CALLISTO, M. Bioindicadores de qualidade de água como ferramenta em estudos de impacto ambiental. Revista FAPAM, v. 2, n. 1, 2003.

HAMMER, O.; HARPER, D. A. T.; RYAN, P. D. PAST: Paleontological statistics software package for education and data analysis. Palaeontologia Electronica, v. 4, 1. Disponível em: <http://palaeo-electronica.org/2001_1/past/ issue1_01.htm. 2001>. Acesso em 15 ago. 2012.

JUNQUEIRA, M. V. et. al. Biomonitoramento da qualidade das águas da Bacia do Alto Rio das Velhas (MG/Brasil) através de macroinvertebrados. Acta Limnologica Brasiliensia, v. 12, p. 73-87, 2000.

KUHLMANN, M. L., IMBIMBO, H. R. V.; WATANABE, H. M. Macrofauna Bentônica De Água Doce: Avanços Metodológicos III. Relatório Técnico. CETESB, 2003. 72 p.

LAKE, P. S.; DOEG, T. J. Macroinvertebrate colonization of stones in two upland southern Australian streams. Hydrobiologia, v. 126, n. 3, p. 199-211, 1985.

LOYOLA, R. G. N. Atual estágio do IAP no uso de índices biológicos de qualidade. In: V SIMPÓSIO DE ECOSSISTEMAS BRASILEIROS: CONSERVAÇÃO, 5., 2000. Vitória. Anais... Vitória: Universidade Federal do Espírito Santo, Vitória, 2000.

MACKAY, R. J. Colonization by lotic macroinvertebrates: a review of processes and patterns. Canadian Journal of Fisheries and Aquatic Sciences, v. 49, p. 617-628, 1992.

MCCAFFERTY, W. P.; PROVONSHA, A. Aquatic entomology: the fishermen's and ecologists' illustrated guide to insects and their relatives. Boston: Science Book International, 1981. $448 \mathrm{p}$. 


\section{MCCUNE, B.; GRACE, J. B. Analysis of Ecological}

Communities. 2. ed. Gleneden Beach: MjM Software, 2002. 304 p.

MEIER, P. G.; PENROSE, D. L.; POLACK, L. The rate of colonization by macroinvertebrates on artificial substrate samplers. Freshwater Biology, v. 9, n. 4, p. 381-392, 1979.

METCALFE, J. L. Biological water quality assessment of running waters based on macroinvertebrates communities: history and present status in Europe. Environmental Pollution, v. 60, p. 101-139, 1989.

MONTEIRO, I. M. et. al. Efeito do tipo de substrato na estrutura da comunidade de macroinvertebrados bentônicos. In: CONGRESSO DE ECOLOGIA DO BRASIL, 8., 2007 Caxambu. Anais... Caxambu, 2007.

MORENO, P.; CALLISTO, M. Indicadores ecológicos: a vida na lama. Ciência Hoje, v. 36, n. 213, p. 68-72, 2005.

MORETTI, M. S. et. al. Invertebrates colonization on native tree leaves in a neotropical stream (Brazil). International

Review of Hydrobiology, v. 92, n. 2, p. 199-210, 2007.

R Core Team. R: A language and environment for statistical computing. R Foundation for Statistical Computing, Vienna, Austria. Disponível em: <URL http://www.R-project.org/>. Acesso em: 15 ago. 2013

ROMANUK, T. N.; LEVINGS, C. D. Associations between arthropods and the supralittoral ecotone: Dependence of aquatic and terrestrial on riparian vegetation. Environmental Entomology, v. 32, n. 6, p. 1343-1353, 2003.

ROSENBERG D. M.; RESH, V. H. The use of artificial substrate in the study of freshwater benthic macroinvertebrates. In: CAIRNS JUNIOR, J. (ed). Artificial substrates. Ann Arbor: Ann Arbor Science/ Butterworth groups, 1982. p. 175-235.

TOWNSEND, C. R.; HILDREW, A. G. Field experiments on the drifting, colonization and continuous redistribution of stream Benthos. Journal of Animal Ecology, v. 45, n. 3, p. $759-772,1976$

WISE, D. H.; MOLLES JUNIOR, M. C. Colonization of artificial substrates by stream insects: influence of substrate size and diversity. Hydrobiologia, v. 65, n. 1, p. 69-74, 1979.
Anexo 1: Tabela de scorers designadas às diferentes famílias de macroinvertebrados aquáticos para a obtenção do índice BMWP.

\begin{tabular}{|c|c|}
\hline Famílias & Scorers \\
\hline $\begin{array}{l}\text { Siphlonuridae, Heptageniidae, Leptophlebiidae, } \\
\text { Potamanthidae, Ephemeridae, Taeniopterygi- } \\
\text { dae, Leuctridae, Capniidae, Perlodidae, Perli- } \\
\text { dae, Chloroperlidae, Aphelocheiridae, Phryga- } \\
\text { neidae, Molannidae, Beraeidae, Odontoceridae, } \\
\text { Leptoceridae, Goeridae, Lepidostomatidae, } \\
\text { Brachycentridae, Sericostomatidae, Calamoce- } \\
\text { ratidae, Helicopsychidae, Megapodagrionidae, } \\
\text { Athericidae, Blephariceridae. }\end{array}$ & 10 \\
\hline $\begin{array}{l}\text { Astacidae, Lestidae, Calopterygidae, Gomphi- } \\
\text { dae, Cordulegastridae, Aeshnidae, Corduliidae, } \\
\text { Libellulidae, Psychomyiidae, Philopotamidae, } \\
\text { Glossosomatidae. }\end{array}$ & 8 \\
\hline $\begin{array}{l}\text { Ephemerellidae, Prosopistomatidae, Nemouri- } \\
\text { dae, Gripopterygidae, Rhyacophilidae, Polycen- } \\
\text { tropodidae, Limnephelidae, Ecnomidae, Hydro- } \\
\text { biosidae, Pyralidae, Psephenidae. }\end{array}$ & 7 \\
\hline $\begin{array}{l}\text { Hydroptilidae, Unionidae, Mycetopodidae, } \\
\text { Hyriidae, Corophilidae, Gammaridae, Hyalelli- } \\
\text { dae, Atyidae, Palaemonidae, Trichodactylidae, } \\
\text { Platycnemididae, Coenagrionidae, Leptohyphi- } \\
\text { dae. }\end{array}$ & 6 \\
\hline $\begin{array}{l}\text { Oligoneuridae, Polymitarcyidae, Dryopidae, } \\
\text { Elmidae (Elminthidae), Helophoridae, Hydro- } \\
\text { chidae, Hydraenidae, Clambidae, Hydropsychi- } \\
\text { dae, Tipulidae, Simuliidae, Planariidae, Den- } \\
\text { drocoelidae, Dugesiidae, Aeglidae. }\end{array}$ & 5 \\
\hline $\begin{array}{l}\text { Baetidae, Caenidae, Haliplidae, Curculionidae, } \\
\text { Chrysomelidae, Tabanidae, Stratyiomyidae, } \\
\text { Empididae, Dolichopodidae, Dixidae, Cerato- } \\
\text { pogonidae, Anthomyidae, Limoniidae, Psycho- } \\
\text { didae, Sciomyzidae, Rhagionidae, Sialidae, } \\
\text { Corydalidae, Piscicolidae, Hydracarina. }\end{array}$ & 4 \\
\hline $\begin{array}{l}\text { Mesoveliidae, Hydrometridae, Gerridae, Ne- } \\
\text { pidae, Naucorid, (Limnocoridae), Pleidae, } \\
\text { Notonectidae, Corixidae, Veliidae, Helodidae, } \\
\text { Hydrophilidae, Hygrobiidae, Dytiscidae, Gyri- } \\
\text { nidae, Valvatidae, Hydrobiidae, Lymnaeidae, } \\
\text { Physidae, Planorbidae, Bithyniidae, Bythinelli- } \\
\text { dae, Sphaeridae, Glossiphonidae, Hirudidae, } \\
\text { Erpobdellidae, Asellidae, Ostracoda. }\end{array}$ & 3 \\
\hline $\begin{array}{l}\text { Chironomidae, Culicidae, Ephydridae, Thau- } \\
\text { maleidae. }\end{array}$ & 2 \\
\hline Oligochaeta (todas as classes), Syrphidae. & 1 \\
\hline
\end{tabular}


Anexo 2: Classes de qualidade e significado dos valores do índice BMWP.

\begin{tabular}{|l|l|l|l|}
\hline Classe & Qualidade & Escore & Significado \\
\hline I & Ótima & $>150$ & $\begin{array}{l}\text { Águas muito limpas } \\
\text { (águas pristinas). }\end{array}$ \\
\hline II & Boa & $121-150$ & $\begin{array}{l}\text { Águas limpas, não } \\
\text { poluídas ou sistema } \\
\text { perceptivelmente não } \\
\text { alterado. }\end{array}$ \\
\hline III & Aceitável & $101-120$ & $\begin{array}{l}\text { Águas muito pouco } \\
\text { poluídas, ou sistema já } \\
\text { com um pouco de alte- } \\
\text { ração. }\end{array}$ \\
\hline IV & Duvidosa & $61-100$ & $\begin{array}{l}\text { São evidentes efeitos } \\
\text { moderados de poluição. }\end{array}$ \\
\hline V & Poluída & $36-60$ & $\begin{array}{l}\text { Águas contaminadas } \\
\text { ou poluídas (sistema } \\
\text { alterado). }\end{array}$ \\
\hline VI & $\begin{array}{l}\text { Muito } \\
\text { Poluída }\end{array}$ & $16-35$ & $\begin{array}{l}\text { Águas muito poluídas } \\
\text { (sistema muito altera- } \\
\text { do). }\end{array}$ \\
\hline VII & $\begin{array}{l}\text { Fortemen- } \\
\text { te Poluída }\end{array}$ & $<36$ & $\begin{array}{l}\text { Águas fortemente polu- } \\
\text { ídas (sistema fortemen- } \\
\text { te alterado). }\end{array}$ \\
\hline \multicolumn{2}{|l|}{ Fonte: http:/www-meioambiente-pr.govbr/modules/conteudo/con- } \\
\hline
\end{tabular}

teudo.php? conteudo $=91$

Recebido em: 22/07/2014

Aceito em: 21/08/2014 